\title{
Understanding the impact of complicated grief on combat related posttraumatic stress disorder, guilt, suicide, and functional impairment in a clinical trial of post-9/11 service members and veterans
}

\author{
Naomi M. Simon MD, MSc ${ }^{1,2}$ | Susanne S. Hoeppner $\operatorname{PhD}^{1,3}$ | Rebecca E. Lubin ${ }^{2}$ (D) \\ Donald J. Robinaugh PhD ${ }^{1,3}$ (i) | Matteo Malgaroli PhD² | Sonya B. Norman PhD ${ }^{4,5,6,7}$ (i) \\ Ron Acierno PhD ${ }^{8,9}$ ( 1 | Elizabeth M. Goetter PhD ${ }^{1,3}$ | Samantha N. Hellberg ${ }^{1}$ | \\ Meredith E. Charney PhD ${ }^{1,3}$ | Eric Bui MD, PhD ${ }^{1,3}$ | Amanda W. Baker PhD ${ }^{1,3}$ | \\ Erin Smith PhD ${ }^{10,11} \mid$ H. Myra Kim ScD ${ }^{10,12}$ (1) Sheila A. M. Rauch PhD ${ }^{13,14}$ (i) \\ ${ }^{1}$ Department of Psychiatry, Massachusetts General Hospital, Boston, Massachusetts \\ ${ }^{2}$ Department of Psychiatry, New York University School of Medicine, New York, New York \\ ${ }^{3}$ Department of Psychiatry, Harvard Medical School, Boston, Massachusetts \\ ${ }^{4}$ National Center for PTSD, White River Junction, Vermont \\ ${ }^{5}$ Mental Health Service Line, Veterans Affairs San Diego Healthcare System, San Diego, California \\ ${ }^{6}$ School of Medicine, University of California, San Diego, California \\ ${ }^{7}$ Veterans Affairs Center of Excellence for Stress and Mental Health, San Diego, California \\ ${ }^{8}$ Mental Health Service Line, Ralph H. Johnson Veterans Affairs Medical Center, Charleston, South Carolina \\ ${ }^{9}$ College of Nursing, Medical University of South Carolina, Charleston, South Carolina \\ ${ }^{10}$ VA Ann Arbor Healthcare System, Ann Arbor, Michigan \\ ${ }^{11}$ Department of Psychiatry, University of Michigan, Ann Arbor, Michigan \\ ${ }^{12}$ Consulting for Statistics, Computing and Analytics Research, University of Michigan, Ann Arbor, Michigan \\ ${ }^{13}$ Mental Health Service Line, Veterans Affairs Atlanta Healthcare System, Decatur, Georgia \\ ${ }^{14}$ Department of Psychiatry and Behavioral Sciences, Emory University School of Medicine, Atlanta, Georgia
}

\section{Correspondence}

Naomi M Simon, MD, MSc, Anxiety and Complicated Grief Program, Department of Psychiatry, One Park Avenue, 8th Floor, New York, NY 10016.

Email: Naomi.Simon@nyulangone.org

Funding information

Medical Research and Materiel Command, Grant/Award Number: W81XWH-11-1-0073; National Center for Advancing Translational Sciences, Grant/Award Number: UL1TR000433

\begin{abstract}
Background: Complicated grief (CG) is a bereavement-specific syndrome distinct from but commonly comorbid with posttraumatic stress disorder (PTSD). While bereavement is common among military personnel (Simon et al., 2018), there is little research on the impact of CG comorbidity on PTSD treatment outcomes.

Methods: To evaluate the impact of comorbid CG on PTSD treatment outcomes we analyzed data from a randomized trial comparing prolonged exposure, sertraline, and their combination in veterans with a primary diagnosis of combat-related PTSD $(n=194)$. Assessment of PTSD, trauma-related guilt, functional impairment, and suicidal ideation and behavior occurred at baseline and weeks 6, 12, and 24 during the 24-week trial. Results: CG was associated with lower PTSD treatment response (odds ratio $(\mathrm{OR})=0.29$, 95\% confidence interval $(\mathrm{Cl})[0.12,0.69], p=0.005)$ and remission (OR $=0.28,95 \% \mathrm{Cl}[0.11,0.71], p=0.007$ ). Those with CG had greater severity of PTSD $(p=0.005)$ and trauma-related guilt $(<0.001)$ at baseline and endpoint. In addition, those
\end{abstract}


with CG were more likely to experience suicidal ideation during the study (CG: 35\%, 14/ 40 vs. no CG 15\%, 20/130; OR = 3.01, 95\% Cl [1.29, 7.02], $p=0.011$ ).

Conclusions: Comorbid CG is associated with elevated PTSD severity and independently associated with poorer endpoint treatment outcomes in veterans with combat-related PTSD, suggesting that screening and additional intervention for CG may be needed.

\section{KEYWORDS}

bereavement, complicated grief, death, military, outcomes, PTSD, treatment

\section{1 | INTRODUCTION}

Loss of a close attachment is among life's greatest stressors (Holmes $\&$ Rahe, 1967). Although the distress and disruption of grief subsides over time for most bereaved adults, approximately $10 \%$ experience a prolonged, impairing grief reaction (Lundorff, Holmgren, Zachariae, Farver-Vestergaard, \& O'Connor, 2017). This grief-related condition was included in the ICD-11 (World Health Organization, 2018) and provisionally in the DSM-5 (American Psychiatric Association, 2013) with different names. Consistent with our prior work and that of many others (e.g., Horowitz et al., 1997; Shear et al., 2011; Simon et al., 2018), we will refer to this syndrome as complicated grief (CG). Although precise diagnostic criteria are still being refined (Mauro et al., 2017; Prigerson \& Maciejewski, 2017; Reynolds, Cozza, \& Shear, 2017), there is broad agreement that core CG symptoms include intense yearning for the deceased, emotional pain, and preoccupation with thoughts about the death or the deceased (American Psychiatric Association, 2013; Prigerson et al., 2009; Simon et al., 2011; World Health Organization, 2018). Individuals with CG may also experience difficulty accepting the death or planning for the future, role and identity confusion, feeling life is unbearable without the deceased, avoidance of reminders of the loss, and in some cases a wish to die to join the deceased (Mauro et al., 2016; Simon et al., 2011).

CG is conceptualized as persistent symptoms of both traumatic distress and separation distress due to the loss of an important attachment (Shear et al., 2011; Simon et al., 2011). Notably, attachment related CG symptoms, such as yearning, are not observed in other disorders that may follow bereavement, such as posttraumatic stress disorder (PTSD) or major depressive disorder (MDD), supporting its designation as a distinct post-loss condition (e.g., Boelen \& van den Bout, 2005; Maercker \& Lalor, 2012; Simon, 2012). Nonetheless, CG often co-occurs with mood, anxiety, and trauma related disorders such as PTSD, and the presence of comorbidity is associated with greater CG severity and greater functional impairment (Kristensen, Weisaeth, Hussain, \& Heir, 2015; Marques et al., 2013; Simon et al., 2007; Sung et al., 2011). CG also shares features with other stressor-related conditions (e.g., PTSD and depression), including emotional distress, avoidance, and guilt (American Psychiatric Association, 2013; Li, Tendeiro, \& Stroebe, 2018).
CG comorbidity with PTSD may be especially relevant for military personnel, given the high risk for exposure to traumatic events as well as significant, sudden, and violent losses (e.g., Toblin et al., 2012; Wisco et al., 2014). A recent study in a military mental health clinic found $43.5 \%$ of outpatients were impacted by a significant loss, nearly half $(46.1 \%)$ of whom exhibited clinically significant CG symptoms (Delaney, Holloway, Miletich, Webb-Murphy, \& Lanouette, 2017). Losses during deployment, especially the loss of a fellow service member (FSM), were associated with greater CG symptom severity than other types of losses. Similarly, in a large sample of post-9/11 treatment-seeking veterans, over $80 \%$ reported a significant loss, and 30\% exhibited clinically significant CG symptoms (Charney et al., 2018). In this sample, CG was associated with more severe PTSD and reduced quality of life. Previous studies of veterans suggest that guilt may play a particularly important role when there is a loss of a FSM, in part due to the nature of the special bonds formed within military units as well as the potential responsibility for decisions that can result in death in combat settings (Lee, Scragg, \& Turner, 2001; Litz et al., 2009; Nazarov et al., 2015). Guilt has also been clearly associated with CG, including feelings of responsibility for the death, guilt about one's behavior toward the deceased in life, or the very fact of living when the deceased person is not (survivor guilt; e.g., see Li et al., 2018).

Our research group recently reported primary results of a randomized controlled trial comparing prolonged exposure (PE), sertraline, and their combination for combat-related PTSD in a large sample of post 9/11 veterans (Rauch, Simon, et al., 2018a, Rauch, $\mathrm{Kim}$, et al., 2018b). Although participants in all three treatment arms improved significantly, no significant difference in PTSD symptom change by treatment assignment was found (Rauch, Simon, et al., 2018a). The correlates of CG comorbidity were examined in this study sample at baseline (Simon et al., 2018). Most participants (79\%) reported a significant death, and $31 \%$ exhibited clinically significant CG symptoms. Notably, those who had lost a FSM (47\%) exhibited a significantly higher rate of CG (41\%) than those reporting other losses (17\%), suggesting this type of loss may be particularly challenging for service members and veterans. CG was associated with greater functional impairment, trauma-related guilt, and lifetime suicide attempts. These data are consistent with prior research supporting an association of CG with functional impairment 
independent of other disorders including PTSD (e.g., Kristensen et al., 2015; Marques et al., 2013). Similarly, suicidal thoughts have been commonly reported in treatment seeking CG samples (Shear et al., 2016; Shear, Wang, Skritskaya, Mauro, \& Ghesquiere, 2014) and in prospective CG research. This includes a study demonstrating an eightfold risk of high suicidal ideation and behaviors even after controlling for depression and PTSD (Latham \& Prigerson, 2004). Given the high prevalence and burden of comorbid CG and PTSD, screening for loss and CG is recommended in military mental health settings, but is not yet routinely implemented (Delaney et al., 2017). This could be, in part, due to our limited understanding of the impact of CG on PTSD outcomes. To our knowledge, no study has examined the impact of comorbid CG symptoms on PTSD treatment outcomes in individuals receiving PE or an antidepressant such as sertraline, both recommended evidence-based treatments for PTSD (e.g., American Psychological Association, 2017; Management of Posttraumatic Stress Disorder Work Group, 2017). Such examination is particularly relevant to military populations, as comorbid PTSD and CG are common and impairing.

The present study thus aimed to examine the impact of cooccurring CG on PTSD treatment outcomes in a sample of post 9/11 veterans with a primary diagnosis of combat-related PTSD. The study data were collected as part of a larger comparative effectiveness trial comparing the efficacy of PE, sertraline, versus their combination in the treatment of PTSD for this population (Rauch, Kim, et al., 2018b), and builds upon the baseline findings reported recently by Simon et al (2018). We hypothesized that comorbid CG would be associated with poorer outcomes as indicated by (a) higher PTSD symptoms, trauma-related guilt, suicidal ideation, and functional impairment at posttreatment, (b) lower PTSD response rate, and (c) lower PTSD remission rates than those without $C G$, even after adjusting for baseline differences in PTSD symptom severity. We further hypothesized that this impact on treatment outcomes would be especially present among those reporting the death of a fellow service member.

\section{2 | METHODS}

\section{1 | Participants}

This study was a planned secondary analysis of a multicenter clinical trial funded by the Department of Defense (W81XWH-11-1-0073). Procedures were approved by site IRBs and DOD Human Research Protection Office (HRPO), and registered at ClinicalTrials.gov (NCT01524133). All participants were randomized to one of three active evidence-based PTSD treatment arms: PE + pill placebo, sertraline (an FDA approved serotonin selective reuptake inhibitor) + an enhanced medication management (EMM) protocol administered by the pharmacotherapist, or PE + sertraline. Detailed methods and primary results have been published (Rauch, Simon, et al., 2018a, Rauch, Kim, et al., 2018b). Briefly, participants were 223 veterans or service members with a primary diagnosis of combat-related PTSD who served in Iraq/Afghanistan wars in the United States military, and 149 completed the 24-week study. The primary modified intentto-treat analyses examined the 207 randomized participants who received the study pills at least once. Our final sample consisted of the subset of the modified intent-to-treat sample who completed a loss cover sheet (indicating the presence or absence of a loss at baseline) and the Inventory of Complicated Grief (ICG; Prigerson et al., 1995: $n=194) ; 13$ subjects (6\%) were excluded for noncompletion.

\section{2 | Measures}

The primary outcome measure was the Clinician-Administered PTSD Scale (CAPS) for DSM-IV (Blake et al., 1995), with diagnosis based on a score of 50 or higher and PTSD present for at least 3 months. The CAPS is commonly used to assess PTSD intensity, frequency, and severity (Blake et al., 1995; Weathers, Keane, \& Davidson, 2001). It has a total score range from 0 to 272 , where higher scores indicate more severe PTSD symptoms. CAPS were administered by raters certified by the standard process of the National Center for PTSD. PTSD treatment response was defined as $\geq 50 \%$ CAPS reduction, and remission was defined as CAPS of 35 or less; all definitions were based on Week 24 or last observed CAPS up to Week 24. Comorbid depression was assessed with the MINI International Neuropsychiatric Interview (MINI) for DSM-IV version 5.0 (Sheehan et al., 1998).

Grief symptoms were measured using the Inventory of Complicated Grief (ICG; Prigerson et al., 1995), a 19-item self-report form with each item rated from 0 (not at all) to 4 (always) that has a total score range of $0-76$, where higher scores indicate greater symptom severity. The ICG was completed only if the participant selected yes to the cover sheet question "Have you had an important person in your life pass away?" The cover sheet included questions about the relation of important lifetime losses (including spouse, parent, child, sibling, grandparent, grandchild, other relative, significant other, partner, fiancé, friend, fellow service member, or other), as well as which death was the most distressing. The ICG was completed in relation to this loss. Threshold CG was defined as an ICG score of 30 or higher (Prigerson et al., 1995) at baseline. The term "CG" is used to represent threshold CG symptoms throughout this paper.

Three additional measures were examined at baseline and endpoint in relation to threshold CG. The Trauma-Related Guilt Inventory (TRGI), a 32-item (each ranging from 0 to 4, where higher scores indicate greater symptom severity) questionnaire measuring cognitive and emotional aspects of guilt associated with the traumatic event (Kubany et al., 1996), comprises three factors: global guilt, distress, and guilt cognitions. The Inventory of Functional Impairment (IFI) is an 80-item assessment of functional impairment related to PTSD in seven domains (romantic relationships, family relationships, work, friendships and socializing, parenting, education, and self-care) rated from $0 \%$ to $100 \%$, with higher scores representing greater impairment (Rodriguez, Holowka, \& Marx, 2012). Finally, the clinician-rated Columbia Suicide Severity Rating Scale (C-SSRS), a standardized 10-point rating assessing a wide spectrum of suicidal ideation (SI) and behaviors (Posner et al., 2008, 2011), was used to assess $\mathrm{SI}$ and behaviors. We examined two C-SSRS outcomes during 
treatment: any SI following baseline ( $n=170$ with post-baseline assessments) and worse SI compared to past-month history at baseline (i.e., new SI or SI worse than baseline; $n=163$ with baseline and at least one postbaseline assessment).

\section{3 | Statistical analyses}

All models were run in SAS version 9.4 for Windows. For categorical outcomes (i.e., treatment response, remission, any and worse SI during treatment), we used logistic regressions with CG at baseline as the main predictor of interest. For continuous outcomes measured over time (i.e., CAPS, TRGI sub-scales, IFI), we used repeated measures mixed models with treatment, time (categorical; baseline, weeks 6, 12, and 24), CG at baseline (yes vs. no), and the interactions of time by treatment and time by CG as predictors. Time was modeled as a repeated measure within participant, using an unstructured covariance matrix to account for the different variances at each visit and different time-steps between visits. Consistent with the primary trial outcome paper (Rauch, Simon, et al., 2018a), all models were adjusted for site and sex, and all models, except for the longitudinal CAPS model, were also adjusted for baseline CAPS. The longitudinal CAPS model retained baseline values as outcomes and allowed groups to differ at baseline. We used specific contrasts to test whether the between CG-group differences observed at baseline changed at Week 24 (end-of-treatment). Means for longitudinal outcomes are presented as model-adjusted least-squares means (LSM) with 95\% confidence intervals, and significance was evaluated with two-sided tests using $\alpha=0.05$. Effect sizes $(d)$ between groups were based on LSM differences divided by the pooled standard deviation of available scores at each time point. In a follow-up model, we split the CG group into those who had indicated their most distressing loss was a fellow service member $(n=25)$ versus those who indicated some other loss $(n=21)$ as most distressing, to examine the effect of type of loss on CAPS treatment outcome. Because there were no significant differences between treatment groups for overall PTSD outcomes (Rauch, Simon, et al., 2018a) and power was limited, we did not plan significance testing of differential CG effects on PTSD outcomes by treatment type.

\section{3 | RESULTS}

CG was present for $24 \%(46 / 194)$ of participants. The CG sample had a mean ICG $(M \pm S D)$ of $40.9 \pm 9.0$. The non-CG sample (148/194) included subjects reporting a loss but no CG $(n=108$ : mean ICG $=16.6 \pm 8.1)$ plus those without a reported loss $(n=40)$. Table 1 describes demographics and baseline clinical symptom severity. There was some missing data for select baseline characteristics reported in Table 1: race and ethnicity (no$C G=2, C G=0$ ), marital status (no-CG $=2, C G=0$ ), service capacity (no$C G=1, C G=0$ ), number of deployments (no-CG =3, CG=0), serviceconnected disability (no-CG $=1, C G=0$ ), lifetime suicidality (no-CG $=6$, $C G=1$ ), and past month suicidality (no-CG $=7, C G=1$ ). Demographics and military service factors did not differ by presence of CG, with the exception of ethnicity; Hispanic participants had a lower proportion of CG (Table 1). The time since the primary loss was 12 or more months before baseline in $83 \%$ of both the CG and non-CG groups. Loss of a FSM was reported as the primary most distressing loss by $56 \%(25 / 46)$ of those with CG, compared to only $27 \%$ (29/108) of those with a loss but no CG. Consistent with our prior baseline report from this study (Simon et al., 2018), baseline CG was associated with greater PTSD severity (CAPS), as well as greater trauma-related guilt (three TRGI subscales) and functional impairment (Table 2). Suicidal ideation and behavior did not vary by CG at baseline (Table 1).

\section{1 | PTSD response and remission}

CG was associated with lower odds of achieving PTSD treatment response (adjusted odds ratio $(A O R)=0.29,95 \% \mathrm{Cl}[0.12,0.69]$, $p=0.005$ ) or remission (AOR $=0.28,95 \% \mathrm{Cl}[0.11,0.71], p=0.007$ ) by Week 24 (end-of-treatment; see Figure 1), after adjusting for site, sex, and baseline CAPS. Response rates for those with comorbid CG compared to no-CG were respectively: 7\% (1/15) versus 33\% (16/49) for PE + placebo, $27 \%(4 / 15)$ versus $47 \%$ (25/53) for sertraline + EMM, and $19 \%(3 / 16)$ versus $46 \%(21 / 46)$ for $P E+$ sertraline.

\section{2 | Symptom change and endpoint severity with PTSD treatment}

The greater symptom severity associated with CG at baseline persisted at treatment endpoint for CAPS severity $(p=0.003$, $d=0.57)$, TRGI global guilt $(p<0.001, d=0.75)$, TRGI distress ( $p=0.025, d=0.43$ ), and TRGI guilt cognitions ( $p=0.004, d=0.53$ ). Functional impairment with CG was greater at baseline $(p=0.033$, $d=0.35$ ), but not at end-of-treatment ( $p=0.324, d=0.18$ ). CG was not associated with a significant difference in the amount of change in CAPS (see Figure 2a), TRGI subscales, or IFI over the 24 weeks (Table 2).

\subsection{Suicidal ideation and CG}

There were no baseline differences in lifetime or past month suicidal ideation (SI) between groups (Table 1). Participants with CG were more likely to experience some level of SI during the 24 weeks of the study (OR: $3.01,95 \% \mathrm{Cl}[1.29,7.02], \mathrm{p}=0.011$ ). There were no differences in the odds of experiencing worse SI during the treatment period relative to the month before treatment (26\% (10/39) vs. $12 \%$ (15/124); OR: $2.54,95 \% \mathrm{Cl}[0.99,6.51], \mathrm{p}=0.053)$. Suicidal behavior during treatment was rare $(3 \%)$ in both groups.

\subsection{Impact of loss of a fellow service member}

To examine the association of CG with PTSD symptom severity in greater detail, we split the sample of participants with CG into those who had CG due to the loss of a FSM (CG-FSM) and those who had CG due to non-FSM losses (CG non-FSM). In contrast to our hypotheses, the CG-FSM sub-sample was more similar to the no-CG 
TABLE 1 Baseline characteristics of treatment seeking post-9/11 combat veterans with and without comorbid complicated grief

\begin{tabular}{|c|c|c|c|}
\hline Outcomes & No CG $(n=148), M / \%(S D / N)$ & CG $(n=46), M / \%(S D / N)$ & $p<0.05$ \\
\hline \multicolumn{4}{|l|}{ Demographics } \\
\hline Age, years, M (SD) & $34.6(8.7)$ & $34(7.4)$ & \\
\hline \multicolumn{4}{|l|}{ Race, \% (n) } \\
\hline White & $61(89)$ & $52(24)$ & \\
\hline Hispanic, \% (n) & $19(28)$ & $4(2)$ & $*$ \\
\hline High school or less, \% ( $n$ ) & $36(53)$ & $37(17)$ & \\
\hline \multicolumn{4}{|l|}{ Marital status, \% (n) } \\
\hline Single, never married & $22(33)$ & $24(11)$ & \\
\hline Widowed & $0(0)$ & $0(0)$ & \\
\hline \multicolumn{4}{|l|}{ Employment, \% ( $n)$} \\
\hline Full-time & $46(68)$ & $61(28)$ & \\
\hline Part-time & $12(18)$ & $11(5)$ & \\
\hline None & $42(62)$ & $28(13)$ & \\
\hline \multicolumn{4}{|l|}{ Service and disability } \\
\hline \multicolumn{4}{|l|}{ Service capacity, most recent, \% (n) } \\
\hline Regular armed forces & $86(127)$ & $89(41)$ & \\
\hline Reserve unit & $3(4)$ & $4(2)$ & \\
\hline Comorbid MDD, \% (n) & $66(98)$ & $78(36)$ & \\
\hline Most distressing loss was a FSM, \% $(n)^{\ddagger}$ & $27(29)$ & $56(25)$ & $* * *$ \\
\hline ICG total score, $M(S D)^{\ddagger}$ & $16.6(8.1)$ & $40.9(9.0)$ & $* * *$ \\
\hline \multicolumn{4}{|l|}{ Suicidality } \\
\hline Any suicidal ideation, lifetime, \% (n) & $39(56)$ & $53(24)$ & \\
\hline Any suicidal ideation, past month, $\%(n)$ & $13(18)$ & $22(10)$ & \\
\hline Any suicidal behavior, lifetime, $\%(n)$ & $10(14)$ & $13(6)$ & \\
\hline Any suicidal behavior, past 3 months, \% (n) & $1(2)$ & $0(0)$ & \\
\hline
\end{tabular}

Abbreviations: CAPS, Clinician-Administered PTSD Scale for DSM-IV; FSM, fellow service-member; ICG, Inventory of Complicated Grief; IFI, Inventory of Functional Impairment; MDD, major depressive disorder; TRGI, Trauma-Related Guilt Inventory (all sub-scales are reported as mean scores).

${ }^{*} p<0.05$.

${ }^{* * *} p<0.001$.

‡Loss type and ICG totals in the no-CG group are based only on those who were bereaved and completed the ICG $(n=108)$.

sample in terms of baseline PTSD symptom severity and symptom change throughout treatment than the CG non-FSM sub-sample (Figure 2b). This appeared to be explained by a greater baseline PTSD symptom severity in the CG non-FSM subsample than the noCG sample $(p=0.004, d=0.69)$, which persisted throughout treatment. Baseline PTSD symptom severity in the CG-FSM subsample (CAPS: $\mathrm{LSM}=62.6,95 \% \mathrm{Cl}[54.8,70.5]$ ) was intermediate between the no-CG sample ( $\mathrm{LSM}=56.0,95 \% \mathrm{Cl}[52.1,60.0])$ and the CG non-FSM subsample (LSM $=74.4,95 \% \mathrm{Cl}[65.5,83.4]$ ), but did not differ significantly from either (CG-FSM vs. no-CG: $p=0.157$, 
TABLE 2 Estimated LSM of PTSD symptom severity, trauma-related guilt severity, and functional impairment at baseline and end-oftreatment (Week 24) in post-9/11 combat veterans with and without comorbid CG

\begin{tabular}{|c|c|c|c|c|c|c|c|c|c|}
\hline \multirow[b]{2}{*}{ Outcome } & \multirow[b]{2}{*}{ Week } & \multicolumn{2}{|l|}{ No CG } & \multicolumn{2}{|l|}{ CG } & \multirow{2}{*}{$\begin{array}{l}\text { Pair-wise } \\
p<0.05\end{array}$} & \multirow[b]{2}{*}{$\mathrm{SD}_{\text {pooled }}$} & \multirow[b]{2}{*}{$d$} & \multirow{2}{*}{$\begin{array}{l}\text { Interactior } \\
\text { contrast } p\end{array}$} \\
\hline & & LSM & $95 \% \mathrm{CL}$ & LSM & $95 \% \mathrm{CL}$ & & & & \\
\hline CAPS total scores & 24 & 42.4 & {$[78.1,87.9]$} & 57.2 & {$[48.4,66.0]$} & 0.003 & 25.8 & 0.57 & \\
\hline TRGI global guilt & 0 & 1.44 & {$[1.17,1.71]$} & 2.34 & {$[1.94,2.74]$} & $<0.001$ & 1.16 & 0.78 & 0.632 \\
\hline TRGI distress & 24 & 1.84 & {$[2.65,3.19]$} & 2.24 & {$[1.90,2.58]$} & 0.025 & 0.94 & 0.43 & \\
\hline \multirow[t]{2}{*}{ TRGI guilt cognition } & 0 & 0.78 & {$[0.61,0.94]$} & 1.22 & {$[0.98,1.46]$} & $<0.001$ & 0.68 & 0.65 & 0.566 \\
\hline & 24 & 0.59 & {$[0.42,0.76]$} & 0.96 & {$[0.70,1.22]$} & 0.004 & 0.70 & 0.53 & \\
\hline IFI total scores & 0 & 40.3 & {$[37.3,43.4]$} & 45.3 & {$[40.7,49.9]$} & 0.033 & 14.3 & 0.35 & 0.417 \\
\hline
\end{tabular}

Note: Estimated marginal means were adjusted for treatment type, site, gender, and (for all models except CAPS total scores) CAPS baseline scores in maximum-likelihood mixed models. The effect size $d$ is based on LSM differences divided by the raw pooled standard deviation (SD pooled). The interaction contrast tests whether the no CG vs. CG group difference observed at baseline significantly differs from that at end of treatment (Week 24).

Abbreviations: CG, complicated grief; $\mathrm{CL}$, confidence interval; LSM, least square mean.

$d=0.30$; CG-FSM vs. CG non-FSM: $p=0.214, d=-0.36$ ). We similarly found no difference in the change in PTSD symptoms between the CG-FSM subsample and the no-CG sample (interaction contrast: $p=0.553$; end-of-treatment difference: $p=0.203, d=0.29$ ); by contrast, the CG non-FSM subsample exhibited a significantly smaller reduction in PTSD symptoms than the no-CG sample (interaction contrast: $p=0.023$; end-of-treatment difference: $p=0.001, d=1.01$ ).

\section{4 | DISCUSSION}

This is the first study to examine the impact of co-occurring CG on PTSD outcomes in those receiving evidence-based PTSD treatment with sertraline, PE, or their combination. As hypothesized, CG was associated with significantly lower odds of PTSD treatment response and remission, after adjustment for baseline PTSD severity (CAPS score) and other covariates. This appeared to be due to those with CG having more severe PTSD at baseline. Thus, while participants with PTSD and comorbid CG had a comparable magnitude of reduction in PTSD symptoms across treatment during the trial, a higher percentage of those with CG nonetheless remained above the threshold for PTSD response or remission at study endpoint. Consistent with this, the CG group had greater posttreatment PTSD symptom severity and greater trauma-related guilt relative to those without CG at endpoint. Further suggesting comorbid CG is a marker of greater severity, those with CG were also more likely to experience suicidal ideation during PTSD treatment compared to those without CG; however, this represented persistence, not worsening, of SI in the CG group. Given these findings, (a)

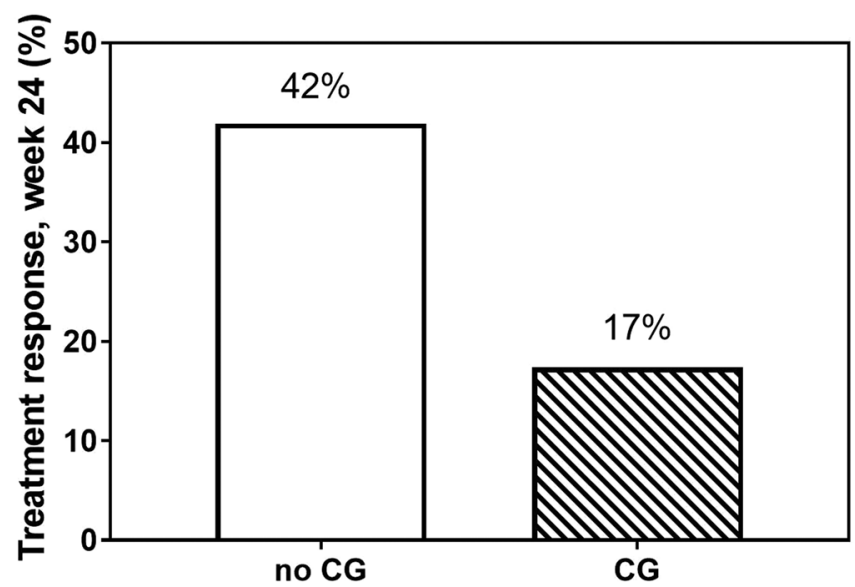

(b)

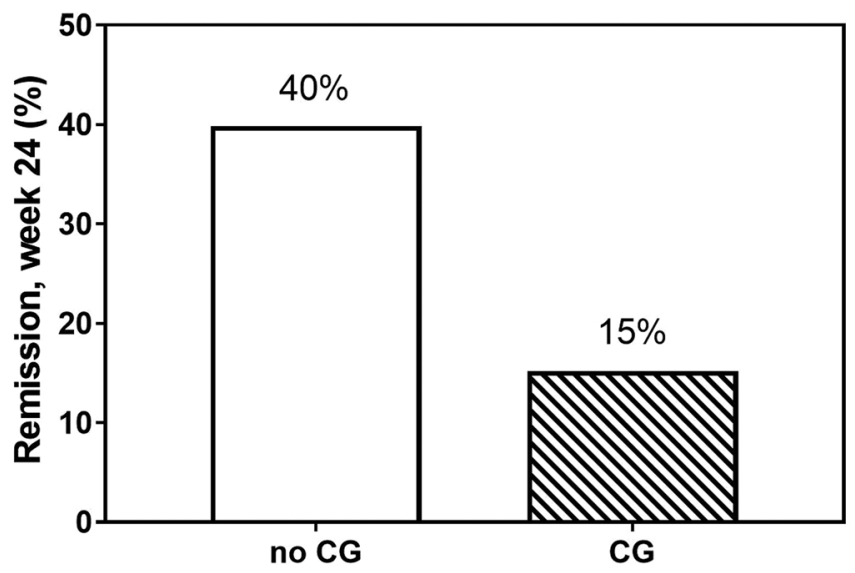

FIGURE 1 Association between baseline comorbid complicated grief and treatment response (panel a) and PTSD remission (panel b) in post9/11 combat veterans at the end of 24 weeks of treatment for PTSD. CG, complicated grief; PTSD, posttraumatic stress disorder 
(a)
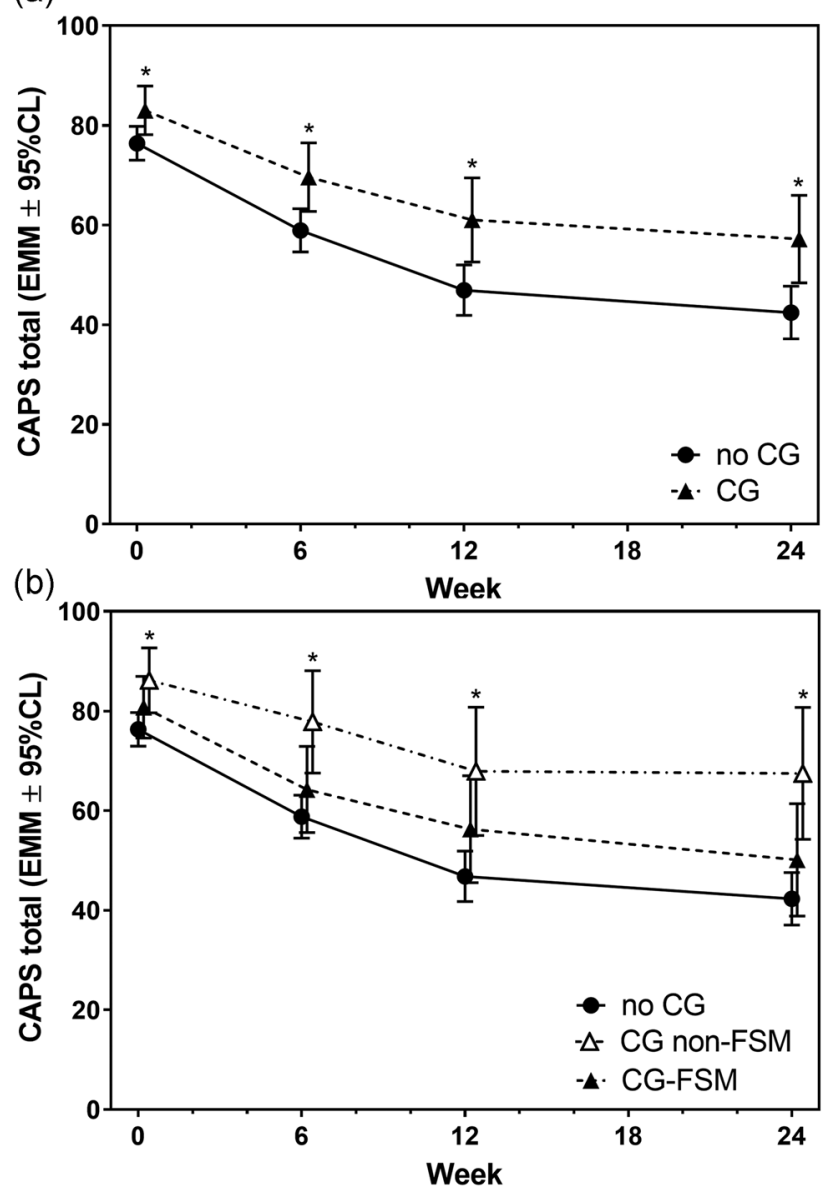

FIGURE 2 Association of baseline comorbid CG with PTSD symptom severity over time in post-9/11 combat veterans in treatment for PTSD. (a) All veterans with CG are combined. (b) Veterans with CG are split into those who indicated that their most distressing loss involved a fellow service member (CG-FSM) and those whose most distressing loss was not a fellow service member (CG non-FSM). Data points for CG groups are nudged to show the confidence intervals more clearly, and stars above the upper confidence limits indicate group differences from that group to the non-CG group $(p<0.05)$. CG, complicated grief; FSM, fellow servicemember; PTSD, posttraumatic stress disorder

it is unclear why CG was not associated with greater endpoint residual functional impairment.

The current sample comprises a post-9/11 combat trauma sample and as such, these findings should be considered in the context of prior military grief research. For example, a study of Vietnam veterans with chronic PTSD suggested that grief is often unaddressed and can persist for over 30 years after a combat loss at levels similar to spousal loss (Pivar \& Field, 2004). Papa et al. (2008) have noted that grief symptoms do not fit in the prevailing fear conditioning models of PTSD, and that combat losses in particular may activate survivor guilt and contribute to suicide risk.

In contrast to our hypotheses and prior reports of the impact of military losses, we found some evidence that CG due to losses that were not a FSM may be associated with even greater PTSD severity and be more likely to impair PTSD treatment response as measured both by the severity of PTSD symptoms at endpoint and the amount of change in CAPS (see Figure 2b). In other words, while our data cannot address this directly, CG due to non-FSM losses could be acting more similarly to a comorbid condition in worsening PTSD severity. Additional research is needed to understand how this may occur, including the effect of non-FSM losses on social support and coping with combat related traumas. The higher rate of PTSD symptom change during treatment seen in veterans with CG due to FSM losses relative to those with CG due to non-FSM losses may be due to a greater overlap between FSM losses and the primary combat trauma (which patients were directly addressing in PTSD treatment). In other words, the effects of therapy based on the index combat trauma may not generalize as well to non-FSM bereavement. Although our sample sizes are too low to perform additional analyses by treatment type, future studies should carefully assess if these potential explanations may be at play for those in PE specifically. Those with additional nonmilitary losses and associated CG symptoms distinct from their index trauma may need additional intervention to achieve optimal PTSD responses. Importantly, our findings do not suggest that the loss of a FSM is not impactful; in contrast, our prior work demonstrated that those with PTSD and the loss of a FSM have higher CG symptom severity and are more likely to cross the symptom threshold for CG than those bereaved due to other causes (Charney et al., 2018; Simon et al., 2018).

It is worth noting that there was a high rate of comorbid major depressive disorder (MDD) in this population overall, and even higher (75\%) in the CG comorbidity group (Table 1). This is consistent with prior research suggesting that after traumatic loss there may be a subgroup of individuals who have high levels of psychiatric comorbidity and associated distress (e.g., Heeke, Stammel, Heinrich, \& Knaevelsrud, 2017; Simon et al., 2007). Future studies should examine the effects of comorbid CG with or without MDD on PTSD as well as on CG treatment outcomes. Additional research on how to optimally treat comorbid CG, PTSD, and MDD after loss and combat trauma is needed. For example, the psychotherapy complicated grief treatment (CGT) has good efficacy for CG despite the high cooccurrence of CG with PTSD and MDD (Shear et al., 2016), but its impact on combat PTSD with comorbid CG is unknown. Overall, more research is needed to understand the optimal treatment approaches for enhancing PTSD outcomes in the presence of CG comorbidity, including whether additional evidence-based treatments for grief (e.g., Shear, Frank, Houck, \& Reynolds, 2005; Shear at al., 2016; Boelen, de Keijser, van den Hout, \& van den Bout, 2007; Bryant et al., 2014) or other combined approaches for comorbidity (e.g., Smid et al., 2015) may be needed in addition to trauma focused work (Papa et al., 2008).

\section{5 | LIMITATIONS}

Our study has some limitations. The presence of CG was based solely on self-report, using an ICG score of 30 or higher; this is the most 
conservative threshold commonly associated with clinical significance though lower thresholds have also been used (American Psychological Association, 2018; Arizmendi, Kaszniak, \& O'Connor, 2016; Kersting, Brahler, Glaesmer, \& Wagner, 2011; Kersting et al., 2009; Ott, 2003; Prigerson et al., 2009; Simon et al., 2007). In addition, because CG was not the primary focus of the parent trial, we do not have detailed information regarding the nature of the death (e.g., suicide, combat) or its possible relation to the key traumatic event reported by participants. We also lack sufficient data to report the effect of PTSD treatments on CG symptom change or its correlation with PTSD treatment response. Further, $17 \%$ of primary losses reported occurred less than 12 months before baseline, suggesting there could be some participants with grief persisting less than 6 months. Future studies should more closely examine the time course of effects and whether time since loss and nature of the death differentially predict PTSD outcomes.

Of interest, while there was some suggestion that there may be differences in the impact of comorbid CG on PTSD outcomes by type of treatment, we were underpowered to test this possibility. Finally, all participants were presenting with a chief complaint of PTSD, not grief related distress; these data do not address how to best treat veterans and service members with primary CG.

\section{6 | CONCLUSIONS}

CG commonly occurs in service members and veterans with combat related PTSD. Our data showed lower response and remission rates in those with comorbid CG relative to those without comorbid CG. In the parent trial, participants in all three treatment arms improved significantly and no significant difference in PTSD symptom change was found by treatment (Rauch, Simon, et al., 2018a). Although our study was not designed to test for differential treatment outcomes by CG, our data suggest lower response and remission rates for all three treatments in those with comorbid CG versus without comorbid CG. Currently, CG is not routinely screened in military treatment settings. Our findings add to the growing evidence that such screening may be warranted (e.g., Currier \& Holland, 2012; Papa et al., 2008; Toblin et al., 2012) along with additional research on how to optimally treat PTSD with CG comorbidity.

\section{ACKNOWLEDGMENTS}

This study was supported by the U.S. Department of Defense through the U.S. Army Medical Research and Materiel Command (MRMC; Randomized Controlled Trial of Sertraline, Prolonged Exposure Therapy, and Their Combination in OEF/OIF Combat Veterans with PTSD); (Award \#W81XWH-11-1-0073; PI: Rauch); the National Center for Advancing Translational Sciences of the National Institutes of Health (Award \#UL1TR000433). This material is the result of work supported with resources and the use of facilities at Massachusetts General Hospital, the VA Ann Arbor Healthcare
System, Ralph H. Johnson VA Medical Center, and VA San Diego Healthcare System.

\section{CONFLICT OF INTERESTS}

Dr. Simon reports grant funding from the American Foundation for Suicide Prevention, DoD, NIH, Janssen, PCORI, and Highland Street Foundation; personal fees from Aptinyx, Springworks, Praxis Therapeutics, Axovant, and MGH Psychiatry Academy; and spousal equity from G1 Therapeutics, outside the submitted work. Dr. Bui receives royalties from Springer Nature for a textbook on grief reactions and for a textbook on anxiety disorders. Dr. Rauch reports royalties from Oxford University Press, outside the submitted work. All other authors report no conflicts of interest.

\section{ORCID}

Naomi M. Simon (D) http://orcid.org/0000-0002-8867-889X Susanne S. Hoeppner (D) http://orcid.org/0000-0003-2060-1666 Rebecca E. Lubin (D) http://orcid.org/0000-0002-1960-8986 Donald J. Robinaugh (D) http://orcid.org/0000-0002-5579-7518 Matteo Malgaroli (D) http://orcid.org/0000-0003-4209-6939 Sonya B. Norman (D) http://orcid.org/0000-0002-4751-1882 Ron Acierno (D) http://orcid.org/0000-0001-8799-8210 Samantha N. Hellberg (D) http://orcid.org/0000-0002-0302-2382 Eric Bui (D) http://orcid.org/0000-0002-1413-6473 Amanda W. Baker (D) http://orcid.org/0000-0002-0103-5411 H. Myra Kim (D) http://orcid.org/0000-0002-0604-8027 Sheila A. M. Rauch (D) http://orcid.org/0000-0001-9686-4011

\section{REFERENCES}

American Psychiatric Association (2013). Diagnostic and statistical manual of mental disorders (5th ed.). Arlington, VA: American Psychiatric Publishing.

American Psychological Association. (2017). Clinical practice guideline for the treatment of posttraumatic stress disorder. Retrieved from: https:// www.apa.org/ptsd-guideline/ptsd.pdf

American Psychological Association. (2018). Inventory of complicated grief (ICG). Retrieved from: https://www.apa.org/pi/about/publications/caregivers/practice-settings/assessment/tools/complicatedgrief.aspx

Arizmendi, B., Kaszniak, A. W., \& O'connor, M. F. (2016). Disrupted prefrontal activity during emotion processing in complicated grief: An fMRI investigation. Neurolmage, 124(Part A), 968-976.

Blake, D. D., Weathers, F. W., Nagy, L. M., Kaloupek, D. G., Gusman, F. D., Charney, D. S., \& Keane, T. M. (1995). The development of a clinicianadministered PTSD scale. Journal of Traumatic Stress, 8, 75-90.

Boelen, P. A., \& van den Bout, J. (2005). Complicated grief, depression, and anxiety as distinct postloss syndromes: A confirmatory factor analysis. American Journal of Psychiatry, 162(11), 2175-2177.

Boelen, P. A., de Keijser, J., van den Hout, M. A., \& van den Bout, J. (2007). Treatment of complicated grief: A comparison between cognitivebehavioral therapy and supportive counseling. Journal of Consulting and Clinical Psychology, 75(2), 277-284.

Bryant, R. A., Kenny, L., Joscelyne, A., Rawson, N., Maccallum, F., Cahill, C., ... Nickerson, A. (2014). Treating prolonged grief disorder: A randomized clinical trial. JAMA Psychiatry, 71(12), 1332-1339. 
Charney, M. E., Bui, E., Sager, J. C., Ohye, B. Y., Goetter, E. M., \& Simon, N. M. (2018). Complicated grief among military service members and veterans who served after September 11, 2001. Journal of Traumatic Stress, 31(1), 157-162.

Currier, J. M., \& Holland, J. M. (2012). Examining the role of combat loss among Vietnam War veterans. Journal of Traumatic Stress, 25, 102-105.

Delaney, E. M., Holloway, K. J., Miletich, D. M., Webb-Murphy, J. A., \& Lanouette, N. M. (2017). Screening for complicated grief in a military mental health clinic. Military Medicine, 182(9), e1751-e1756.

Heeke, C., Stammel, N., Heinrich, M., \& Knaevelsrud, C. (2017). Conflictrelated trauma and bereavement: Exploring differential symptom profiles of prolonged grief and posttraumatic stress disorder. BMC Psychiatry, 17(1), 118.

Holmes, T. H., \& Rahe, R. H. (1967). The social readjustment rating scale. Journal of Psychosomatic Research, 11(2), 213-218.

Horowitz, M. J., Siegel, B., Holen, A., Bonanno, G. A., Milbrath, C., \& Stinson, C. H. (1997). Diagnostic criteria for complicated grief disorder. American Journal of Psychiatry, 154(7), 904-910.

Kersting, A., Brahler, E., Glaesmer, H., \& Wagner, B. (2011). Prevalence of complicated grief in a representative population-based sample. Journal of Affective Disorders, 131, 339-343.

Kersting, A., Kroker, K., Horstmann, J., Ohrmann, P., Baune, B. T., Arolt, V., ... Suslow, T. (2009). Complicated grief in patients with unipolar depression. Journal of Affective Disorders, 118, 201-204.

Kristensen, P., Weisaeth, L., Hussain, A., \& Heir, T. (2015). Prevalence of psychiatric disorders and functional impairment after loss of a family member: A longitudinal study after the 2004 Tsunami. Depression and Anxiety, 32(1), 49-56.

Kubany, E. S., Haynes, S. N., Abueg, F. R., Manke, F. P., Brennan, J. M., \& Stahura, C. (1996). Development and validation of the trauma-related guilt inventory (TRGI). Psychological Assessment, 8, 428-444.

Latham, A. E., \& Prigerson, H. G. (2004). Suicidality and bereavement: Complicated grief as psychiatric disorder presenting greatest risk for suicidality. Suicide and Life-Threatening Behavior, 34(4), 350-362.

Lee, D. A., Scragg, P., \& Turner, S. (2001). The role of shame and guilt in traumatic events: A clinical model of shame-based and guilt-based PTSD. British Journal of Medical Psychology, 74(4), 451-466.

Li, J., Tendeiro, J. N., \& Stroebe, M. (2018). Guilt in bereavement: Its relationship with complicated grief and depression. International Journal of Psychology, https://doi.org/10.1002/ijop.12483

Litz, B. T., Stein, N., Delaney, E., Lebowitz, L., Nash, W. P., Silva, C., \& Maguen, S. (2009). Moral injury and moral repair in war veterans: A preliminary model and intervention strategy. Clinical Psychology Review, 29, 695-706.

Lundorff, M., Holmgren, H., Zachariae, R., Farver-Vestergaard, I., \& O'Connor, M. (2017). Prevalence of prolonged grief disorder in adult bereavement: A systematic review and meta-analysis. Journal of Affective Disorders, 212, 138-149.

Maercker, A., \& Lalor, J. (2012). Diagnostic and clinical considerations in prolonged grief disorder. Dialogues in clinical neuroscience, 14(2), 167-176.

Management of Posttraumatic Stress Disorder Work Group (2017). VA/ DoD clinical practice guideline for the management of posttraumatic stress disorder and acute stress disorder. Washington, DC: Department of Veterans Affairs and Department of Defense. Version 3.0

Marques, L. M., Bui, E., LeBlanc, N., Porter, E., Robinaugh, D., Dryman, M. T., ... Simon, N. (2013). Complicated grief symptoms in anxiety disorders: Prevalence and associated impairment. Depression and Anxiety, 30, 1211-1216.

Mauro, C., Shear, M. K., Reynolds, C. F., Simon, N. M., Zisook, S., Skritskaya, N., ... Glickman, K. (2016). Performance characteristics and clinical utility of diagnostic criteria proposals in bereaved treatmentseeking patients. Psychological Medicine, 47, 608-615.
Mauro, C., Shear, M. K., Reynolds, C. F., Simon, N. M., Zisook, S., Skritskaya, N., ... Glickman, K. (2017). Performance characteristics and clinical utility of diagnostic criteria proposals in bereaved treatmentseeking patients. Psychological Medicine, 47(4), 608-615.

Nazarov, A., Jetly, R., McNeely, H., Kiang, M., Lanius, R., \& McKinnon, M. C. (2015). Role of morality in the experience of guilt and shame within the armed forces. Acta Psychiatrica Scandinavica, 132, 4-19.

Ott, C. H. (2003). The impact of complicated grief on mental and physical health at various points in the bereavement process. Death Studies, 27, 249-272.

Papa, A., Neria, Y., \& Litz, B. (2008). Traumatic bereavement in war veterans. Psychiatric Annals, 38(10), 686-691.

Pivar, I. L., \& Field, N. P. (2004). Unresolved grief in combat veterans with PTSD. Journal of Anxiety Disorders, 18, 745-755.

Posner, K., Brent, D., Lucas, C., Gould, M., Stanley, B., Brown, G., ... Oquendo, M. (2008). Columbia-suicide severity rating scale (C-SSRS). New York: Columbia University Medical Center.

Posner, K., Brown, G. K., Stanley, B., Brent, D. A., Yershova, K. V., Oquendo, M. A., ... Mann, J. J. (2011). The Columbia-suicide severity rating scale: Initial validity and internal consistency findings from three multisite studies with adolescents and adults. The American Journal of Psychiatry, 168, 1266-1277.

Prigerson, H. G., Horowitz, M. J., Jacobs, S. C., Parkes, C. M., Aslan, M., Goodkin, K., ... Maciejewski, P. K. (2009). Prolonged grief disorder: Psychometric validation of criteria proposed for DSM-V and ICD-11. PLOS medicine, 6, e1000121.

Prigerson, H. G., \& Maciejewski, P. K. (2017). Rebuilding consensus on valid criteria for disordered grief. JAMA Psychiatry, 74(4), 435-436.

Prigerson, H. G., Maciejewski, P. K., Reynolds, C. F., 3rd, Bierhals, A. J., Newsom, J. T., Fasiczka, A., ... Miller, M. (1995). Inventory of complicated grief: A scale to measure maladaptive symptoms of loss. Psychiatry Research, 59, 65-79.

Rauch, S. A. M., Kim, H. M., Powell, C., Tuerk, P. W., Simon, N. M., Acierno, R., ... Hoge, C. W. (2018b). Efficacy of prolonged exposure therapy, sertraline hydrochloride, and their combination among combat veterans with posttraumatic stress disorder: A randomized clinical trial. JAMA Psychiatry, 76, 117. https://doi.org/10.1001/jamapsychiatry.2018.3412

Rauch, S. A. M., Simon, N. M., Kim, H. M., Acierno, R., King, A. P., Norman, S. B., ... Hoge, C. W. (2018a). Integrating biological treatment mechanisms into randomized clinical trials: Design of PROGrESS (PROlonGed ExpoSure and Sertraline Trial). Contemporary Clinical Trials, 64, 128-138.

Reynolds, C. F., Cozza, S. , J., \& Shear, M. K. (2017). Clinically relevant criteria for a persistent impairing grief disorder: Putting patients first. JAMA Psychiatry, 74(5), 433-434.

Rodriguez, P., Holowka, D. W., \& Marx, B. P. (2012). Assessment of posttraumatic stress disorder-related functional impairment: A review. Journal of Rehabilitation Research and Development, 49, 649-665.

Shear, K., Frank, E., Houck, P. R., \& Reynolds, C. F. (2005). Treatment of complicated grief: A randomized controlled trial. Journal of the American Medical Association, 293(21), 2601-2608.

Shear, M. K., Reynolds, C. F., Simon, N. M., Zisook, S., Wang, Y., Mauro, C., ... Skritskaya, N. (2016). Optimizing treatment of complicated grief: A randomized controlled trial. JAMA Psychiatry, 73(7), 685-694.

Shear, M. K., Simon, N., Wall, M., Zisook, S., Neimeyer, R., Duan, N., ... Keshaviah, A. (2011). Complicated grief and related bereavement issues for DSM-5. Depression and Anxiety, 28, 103-117.

Shear, M. K., Wang, Y., Skritskaya, N., Mauro, C., \& Ghesquiere, A. (2014). Treatment of complicated grief in elderly persons: A randomized clinical trial. JAMA Psychiatry, 71(11), 1287-1295.

Sheehan, D. V., Lecrubier, Y., Sheehan, K. H., Amorim, P., Janavs, J., Weiller, E., ... Dunbar, G. C. (1998). The Mini-International Neuropsychiatric Interview (M.I.N.I): The development and validation of a structured diagnostic psychiatric interview for DSM-IV and ICD-10. Journal of Clinical Psychiatry, 59(20), 22-33. 
Simon, N. M. (2012). Is complicated grief a post-loss stress disorder? Depression and Anxiety, 29(7), 541-544.

Simon, N. M., O'Day, E. B., Hellberg, S. N., Hoeppner, S. S., Charney, M. E., Robinaugh, D. J., ... Rauch, S. A. M. (2018). The loss of a fellow service member: Complicated grief in post-9/11 service members and veterans with combat-related posttraumatic stress disorder. Journal of Neuroscience Research, 96, 5-15.

Simon, N. M., Shear, K. M., Thompson, E. H., Zalta, A. K., Perlman, C., Reynolds, C. F., ... Silowash, R. (2007). The prevalence and correlates of psychiatric comorbidity in individuals with complicated grief. Comprehensive Psychiatry, 48(5), 395-399.

Simon, N. M., Wall, M. M., Keshaviah, A., Dryman, M. T., LeBlanc, N. J., \& Shear, M. K. (2011). Informing the symptom profile of complicated grief. Depression and Anxiety, 28, 118-126.

Smid, G. E., Kleber, R. J., de la Rie, S., Bos, J. B. A., Gersons, B. P. R., \& Boelen, P. A. (2015). Brief Eclectic Psychotherapy for Traumatic Grief (BEP-TG): Toward integrated treatment of symptoms related to traumatic loss. European Journal of Psychotraumatology, 6(1), 27324.

Sung, S. C., Dryman, M. T., Marks, E., Shear, M. K., Ghesquiere, A., Fava, M., ... Simon, N. M. (2011). Complicated grief among individuals with major depression: Prevalence, comorbidity, and associated features. Journal of Affective Disorders, 134(1-3), 453-458.
Toblin, R. L., Riviere, L. A., Thomas, J. L., Adler, A. B., Kok, B. C., \& Hoge, C. W. (2012). Grief and physical health outcomes in US soldiers returning from combat. Journal of Affective Disorders, 136, 469-475.

Weathers, F. W., Keane, T. M., \& Davidson, J. R. (2001). Clinicianadministered PTSD scale: A review of the first ten years of research. Depression and Anxiety, 13, 132-156.

Wisco, B. E., Marx, B. P., Wolf, E. J., Miller, M. W., Southwick, S. M., \& Pietrzak, R. H. (2014). Posttraumatic stress disorder in the US veteran population: Results from the National Health and Resilience in Veterans Study. The Journal of Clinical Psychiatry, 75, 1338-1346.

World Health Organization (2018). International classification of diseases $11^{\text {th }}$ revision (ICD-11). Geneva: World Health Organization.

How to cite this article: Simon NM, Hoeppner SS, Lubin RE, et al. Understanding the impact of complicated grief on combat related posttraumatic stress disorder, guilt, suicide, and functional impairment in a clinical trial of post-9/11 service members and veterans. Depress Anxiety. 2020;37: 63-72. https://doi.org/10.1002/da.22911 